\title{
Multi-UAVs Cooperative Localization Algorithms with Communication Constraints
}

\author{
Xiaowei Fu, ${ }^{1}$ Haiyang Bi, ${ }^{2}$ and Xiaoguang Gao ${ }^{1}$ \\ ${ }^{1}$ School of Electronics and Information, Northwestern Polytechnical University, Xian, Shaanxi 710129, China \\ ${ }^{2}$ Shenyang Aircraft Design and Research Institute, Shenyang, Liaoning 110035, China
}

Correspondence should be addressed to Xiaowei Fu; fxw@nwpu.edu.cn

Received 4 June 2017; Revised 31 July 2017; Accepted 3 August 2017; Published 7 September 2017

Academic Editor: Filippo Cacace

Copyright (C) 2017 Xiaowei Fu et al. This is an open access article distributed under the Creative Commons Attribution License, which permits unrestricted use, distribution, and reproduction in any medium, provided the original work is properly cited.

Communication is the basis of multi-UAVs cooperative localization. There will be some communication delay and packet loss when one UAV communicates with others, and these communication restrictions may have negative influences on multi-UAV cooperative localization. In this paper, the communication among UAVs is described as Bernoulli random variables, and two kinds of cooperative localization algorithms are proposed. One is centralized algorithm, where a kind of one-step prediction strategy is designed. The other is distributed algorithm, where a kind of prediction-compensation strategy is proposed and weighted expectation state estimation method is designed. Simulation results show the effectiveness of the proposed algorithms.

\section{Introduction}

In the future, air-to-ground attack and target indication will be important tasks for UAVs, and targets localization accuracy is the key factor to influence whether UAVs can accomplish these tasks or not. Currently, the single station targeting method is often used by UAVs, but multi-UAVs cooperative positioning can improve localization accuracy. Communication is the basis of multi-UAVs cooperative positioning. However, there always are some communication latency and information packet loss when UAVs communicate with each other, and these communication restrictions may have negative effect on multi-UAVs cooperative localization.

In order to eliminate the negative effects of communication delay and packet loss, it is necessary to present some information fusion mechanisms to improve multiUAVs cooperative positioning accuracy. A kind of suboptimal fusion estimation method is proposed in [1] to resolve the random packet loss problem of centralized multiple sensors information fusion. Literature [2] proposed a method of optimal estimation to resolve the problem of random communication latency packet loss. Literature [3] presents a three-period distributed Kalman fusion estimation strategy for wireless sensor networks random packet losses problem. Literature [4] is concerned with the distributed fusion estimation problem for discrete-time stochastic linear system with multiple delay. Literature [5] uses a predicted modal to describe communication latency, and a kind of suboptimal $\mathrm{H}$-filter is designed. A kind of centralized $\mathrm{H}$-filter is designed to resolve fusion problem with communication latency, data out-of-order, and packet loss in [6].

All this work is based on the fact that there was prior information about communication delay and packet loss. But in most situations, it is hard to get this information in advance. Two methods are proposed to describe communication latency and packet loss. One is Markov chain in [7]; the other is random variable with Bernoulli distribution in [8-10].

In this paper, the communication latency and packet loss among UAVs are described as Bernoulli random variables, and two kinds of cooperative localization algorithms are proposed. One is centralized algorithm, where a kind of onestep prediction strategy is designed. The other is distributed algorithm, where a kind of prediction-compensation strategy is proposed and weighted expectation state estimation 


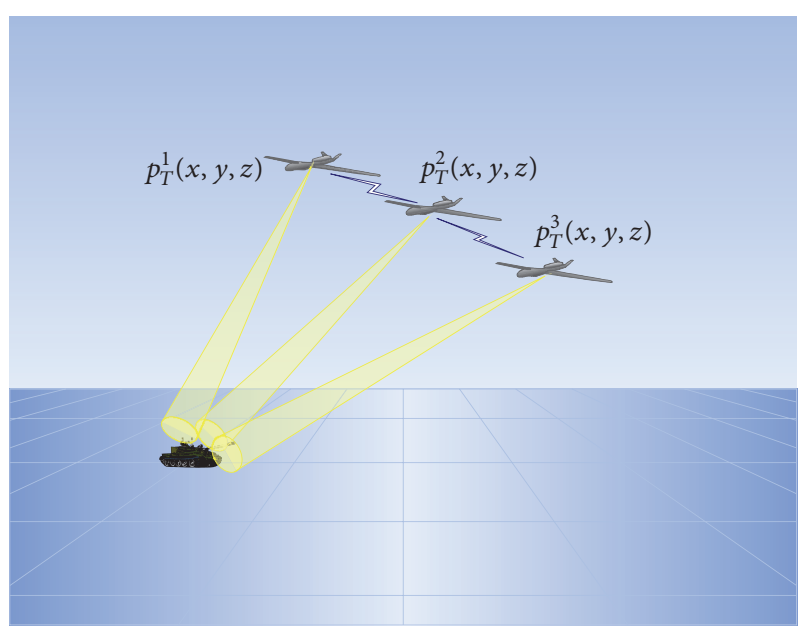

FIGURE 1: Multi-UAVs cooperative Localization.

method is designed. Simulation results show the effectiveness of the proposed algorithms.

\section{Description of Multi-UAVs Cooperative Localization Problem}

In Figure 1, multiple UAVs are detecting the target independently. One UAV is defined as the fusion center, which is the leader of multi-UAVs; other UAVs are followers. Each UAV gets target location information by passive sensor; then the positioning information is transmitted to the fusion center. The leader fuses this information to obtain accurate positioning information.

Packet loss and communication latency in the process of communication have negative influence on the precision of UAV's cooperative localization. Packet loss leads to the fact that fusion center cannot receive complete data packet from followers, and communication delay leads to the fact that the leader cannot receive data packet from followers synchronously. All these result in the fact that the leader cannot obtain accurate target position.

Every follower communicates with the leader directly. There will be packet loss and time delay in the process of communication, and there was no prior information about information delay and packet loss. There only was the possibility of packet loss and time delay.

\section{Multi-UAVs Cooperative Passive Localization}

Based on the description of multi-UAVs cooperative localization problem, this section presents two kinds of multi-UAVs cooperative passive localization algorithms under communication constraints, including centralized and distributed algorithm.

3.1. Centralized Multi-UAVs Cooperative Localization Algorithm. In this algorithm, every follower sends its sensed information to the leader without processing, and the leader fuses all this received information to obtain target position.

We use the following state-space model to describe target's motion and communication constraints

$$
\begin{aligned}
\mathbf{x}_{k+1}= & \boldsymbol{\Phi}_{k} \mathbf{x}_{k}+\boldsymbol{\Gamma}_{k} \mathbf{w}_{k}, \\
\mathbf{z}_{k}^{i}= & \mathbf{H}_{k}^{i} x_{k}+\mathbf{v}_{k}^{i}, \\
\mathbf{y}_{k}^{i}= & \xi_{1}^{i}(k) \mathbf{z}_{k}^{i}+\xi_{2}^{i}(k) \mathbf{z}_{k-1}^{i} \\
& +\left(1-\xi_{1}^{i}(k)-\xi_{2}^{i}(k)\right) \mathbf{y}_{k-1}^{i},
\end{aligned}
$$

where $\mathbf{x}_{k}$ represents the target position state at time $k, \mathbf{z}_{k}^{i}$ represents target position information that is sensed by the $i$ th UAV at time $k, \mathbf{y}_{k}^{i}$ represents target position information that is received by the leader from $i$ th UAV at time $k, \Phi_{k}$ represents system state transition matrix, $\Gamma_{k}$ represents system noise matrix, $\mathbf{H}_{k}^{i}$ represents the $i$ th UAV's measurement matrix at time $k, \mathbf{v}_{k}^{i}$ represents measurement noise of $i$ th UAV at time $k$, and $\mathbf{w}_{k}$ represents system noise.

In formula (3), variable $\xi_{1}^{i}(k)$ and $\xi_{2}^{i}(k)$ are used to describe the communication situation between fusion center and other UAVs, and these variables obey the Bernoulli distribution. If the $i$ th UAV is the leader, then $\xi_{1}^{i}(k)=1$ represents the fusion center received from the leader which has no packet loss or time delay. If the $i$ th UAV is a follower, $\xi_{2}^{i}(k)$ depends on whether the fusion center receives message from the follower. If the fusion center receives target position without time delay, $\xi_{1}^{i}(k)=1$ and $y_{k}^{i}=z_{k}^{i}$; if the fusion center receives target position with time delay, $\xi_{2}^{i}(k)=1$ and $y_{k}^{i}=z_{k-1}^{i}$; if the fusion center does not receive target position at time $k$, which means packet is lost, then $\xi_{1}^{i}(k)=0$ and $\xi_{2}^{i}(k)=0$.

From formulas (2) and (3), we can get the result as follows:

$$
\begin{aligned}
\mathbf{y}_{k}^{i}= & \xi_{1}^{i}(k)\left(\mathbf{H}_{k}^{i} \mathbf{x}_{k}+\mathbf{v}_{k}^{i}\right)+\xi_{2}^{i}(k) \mathbf{z}_{k-1}^{i} \\
& +\left(1-\xi_{1}^{i}(k)-\xi_{2}^{i}(k)\right) \mathbf{y}_{k-1}^{i} \\
= & \xi_{1}^{i}(k) \mathbf{H}_{k}^{i} \mathbf{x}_{k}+\xi_{2}^{i}(k) \mathbf{z}_{k-1}^{i} \\
& +\left(1-\xi_{1}^{i}(k)-\xi_{2}^{i}(k)\right) \mathbf{y}_{k-1}^{i}+\xi_{1}^{i}(k) \mathbf{v}_{k}^{i} .
\end{aligned}
$$

A new positioning system is constructed by using extended Kalman filter:

$$
\begin{aligned}
\widetilde{\mathbf{x}}_{k+1} & =\widetilde{\boldsymbol{\Phi}}_{k} \widetilde{x}_{k}+\widetilde{\boldsymbol{\Gamma}}_{k} \mathbf{w}_{k}, \\
\widetilde{\mathbf{y}}_{k} & =\widetilde{\mathbf{H}}_{k} \widetilde{\mathbf{x}}_{k}+\boldsymbol{\xi}_{1}(k) \widetilde{\mathbf{v}}_{k} .
\end{aligned}
$$


In formula (5)

$$
\widetilde{\mathbf{x}}_{k}=\left[\begin{array}{c}
\mathbf{x}_{k} \\
\mathbf{z}_{k-1} \\
\mathbf{y}_{k-1}
\end{array}\right],
$$$$
\mathbf{W}_{k}=\left[\begin{array}{l}
\mathbf{w}_{k} \\
\mathbf{V}_{k}
\end{array}\right] \text {, }
$$$$
\mathbf{V}_{k}=\left[\begin{array}{c}
\mathbf{v}_{k}^{1} \\
\vdots \\
\mathbf{v}_{k}^{L}
\end{array}\right],
$$$$
\mathbf{z}_{k}=\left[\begin{array}{c}
\mathbf{z}_{k}^{1} \\
\vdots \\
\mathbf{z}_{k}^{L}
\end{array}\right],
$$$$
\mathbf{y}_{k}=\left[\begin{array}{c}
\mathbf{y}_{k}^{1} \\
\vdots \\
\mathbf{y}_{k}^{L}
\end{array}\right],
$$$$
\mathbf{H}_{k}=\left[\begin{array}{c}
\mathbf{H}_{k}^{1} \\
\vdots \\
\mathbf{H}_{k}^{L}
\end{array}\right],
$$

$$
\begin{aligned}
& \widetilde{\boldsymbol{\Phi}}_{k}=\left[\begin{array}{ccc}
\boldsymbol{\Phi}_{k} & 0 & 0 \\
\mathbf{H}_{k} & 0 & 0 \\
\boldsymbol{\xi}_{1}(k) \mathbf{H}_{k} & \boldsymbol{\xi}_{2}(k) & \mathbf{I}_{m}-\boldsymbol{\xi}_{1}(k)-\boldsymbol{\xi}_{2}(k)
\end{array}\right], \\
& \widetilde{\boldsymbol{\Gamma}}_{k}=\left[\begin{array}{cc}
\boldsymbol{\Gamma}_{k} & 0 \\
0 & \mathbf{I}_{m} \\
0 & \boldsymbol{\xi}_{1}(k)
\end{array}\right], \\
& m=\sum_{i=1}^{L} m^{(i)},
\end{aligned}
$$

$$
\begin{aligned}
& \widetilde{\mathbf{H}}_{k}=\left[\begin{array}{lll}
\boldsymbol{\xi}_{1}(k) \mathbf{H}_{k} & \boldsymbol{\xi}_{2}(k) & \mathbf{I}_{m}-\boldsymbol{\xi}_{1}(k)-\boldsymbol{\xi}_{2}(k)
\end{array}\right], \\
& \boldsymbol{\xi}_{1}(k)=\operatorname{diag}\left[\xi_{1}^{i}(k) \mathbf{I}_{m^{(1)}}, \ldots, \xi_{1}^{i}(k) \mathbf{I}_{m^{(L)}}\right], \\
& \boldsymbol{\xi}_{2}(k)=\operatorname{diag}\left[\xi_{2}^{i}(k) \mathbf{I}_{m^{(1)}}, \ldots, \xi_{2}^{L}(k) \mathbf{I}_{m^{(L)}}\right]
\end{aligned}
$$

where $i$ represents the identification number of UAV, the leader UAV's identification number is $1, L$ represents the quantity of UAVs, and $\operatorname{diag}(\bullet)$ represents the diagonal matrix which diagonal variable is $\bullet$, while $\mathbf{I}_{m}$ is $m \times m$ unit matrix.
The system which formula (5) represented has the noise statistics information as follows:

$$
\begin{aligned}
\mathbf{Q}_{w} & =E\left[\mathbf{W}(t) \mathbf{W}^{\mathrm{T}}(K)\right] \\
& =E\left\{\left[\begin{array}{l}
\mathbf{w}(t) \\
\mathbf{V}(t)
\end{array}\right]\left[\mathbf{w}^{\mathrm{T}}(k), \mathbf{V}^{\mathrm{T}}(k)\right]\right\}=\left[\begin{array}{cc}
\mathbf{Q}_{k} & 0 \\
0 & \mathbf{Q}_{v}
\end{array}\right], \\
\mathbf{Q}_{v} & =E\left[\mathbf{V}(t) \mathbf{V}^{\mathrm{T}}(K)\right]=(R)_{m}, \\
\mathbf{S} & =E\left[\mathbf{W}(t) \mathbf{V}^{\mathrm{T}}(K)\right]=E\left\{\left[\begin{array}{l}
\mathbf{w}(t) \\
\mathbf{V}(t)
\end{array}\right] \mathbf{V}^{\mathrm{T}}(t)\right\} \\
& =\left[\begin{array}{c}
0 \\
\mathbf{Q}_{v}
\end{array}\right],
\end{aligned}
$$

where $\xi_{1}^{i}(k)$ and $\xi_{2}^{i}(k)$ obey the Bernoulli distribution, $\operatorname{prob}\left(\xi_{1}^{i}(k)=1\right)=\alpha_{1}^{i}, \operatorname{prob}\left(\xi_{2}^{i}(k)=1\right)=\alpha_{2}^{i}, 0 \leq \alpha_{1}^{i} \leq 1$, and $0 \leq \alpha_{2}^{i} \leq 1, i=1,2, \ldots, L$, while $\alpha_{1}^{i}$ represents the probability of fusion center receiving position without time delay. $\alpha_{2}^{i}$ represents the probability of fusion center receiving position with time delay.

$\xi^{i}(k)$ obeys the Bernoulli distribution, so that $E\left[\xi^{i}(k)\right]=$ $\alpha^{i}$ and $E\left[\left(\xi^{i}\right)^{2}(k)\right]=\alpha^{i}, i, j=1,2, \ldots, L . E[\bullet]$ represents the mathematical expectation of $\bullet$.

Based on the feature of the Bernoulli distribution, for above system, we can get the conclusion as follows:

$$
\begin{aligned}
& \overline{\boldsymbol{\Phi}}_{k}=E\left[\widetilde{\boldsymbol{\Phi}}_{k}\right]=\left[\begin{array}{ccc}
\boldsymbol{\Phi}_{k} & 0 & 0 \\
\mathbf{H}_{k} & 0 & 0 \\
\boldsymbol{\alpha}_{1} \mathbf{H}_{k} & \boldsymbol{\alpha}_{2} & \mathbf{I}_{m}-\boldsymbol{\alpha}_{1}-\boldsymbol{\alpha}_{2}
\end{array}\right], \\
& \overline{\mathbf{H}}_{k}=E\left[\widetilde{\mathbf{H}}_{k}\right]=\left[\begin{array}{lll}
\boldsymbol{\alpha}_{1} \mathbf{H}_{k} & \boldsymbol{\alpha}_{2} & \mathbf{I}_{m}-\boldsymbol{\alpha}_{1}-\boldsymbol{\alpha}_{2}
\end{array}\right], \\
& \overline{\boldsymbol{\Gamma}}_{k}=E\left[\widetilde{\boldsymbol{\Gamma}}_{k}\right]=\left[\begin{array}{cc}
\boldsymbol{\Gamma}_{k} & 0 \\
0 & \mathbf{I}_{m} \\
0 & \boldsymbol{\alpha}_{1}
\end{array}\right] \text {, }
\end{aligned}
$$

where

$$
\begin{aligned}
& \boldsymbol{\alpha}_{1}=E\left[\boldsymbol{\xi}_{1}(k)\right]=\operatorname{diag}\left(\alpha_{1}^{1} \mathbf{I}_{m_{1}}, \ldots, \alpha_{1}^{L} \mathbf{I}_{m_{L}}\right), \\
& \boldsymbol{\alpha}_{2}=E\left[\boldsymbol{\xi}_{2}(k)\right]=\operatorname{diag}\left(\alpha_{2}^{1} \mathbf{I}_{m_{1}}, \ldots, \alpha_{2}^{L} \mathbf{I}_{m_{L}}\right) .
\end{aligned}
$$

And then

$$
\begin{aligned}
\Delta \boldsymbol{\Phi}_{k} & =\widetilde{\boldsymbol{\Phi}}_{k}-\overline{\boldsymbol{\Phi}}_{k} \\
& =\left[\begin{array}{llc}
0 & 0 & \left(\boldsymbol{\xi}_{1}(k)-\boldsymbol{\alpha}_{1}\right) \mathbf{H}_{k} \\
0 & 0 & \boldsymbol{\xi}_{2}(k)-\boldsymbol{\alpha}_{2} \\
0 & 0 & \left(\boldsymbol{\alpha}_{1}-\boldsymbol{\xi}_{1}(k)\right)+\left(\boldsymbol{\alpha}_{2}-\boldsymbol{\xi}_{2}(k)\right)
\end{array}\right]^{\mathrm{T}}, \\
\Delta \mathbf{H}_{k}= & \widetilde{\mathbf{H}}_{k}-\overline{\mathbf{H}}_{k}=\left[\begin{array}{c}
\left(\boldsymbol{\xi}_{1}(k)-\boldsymbol{\alpha}_{1}\right) \mathbf{H}_{k} \\
\boldsymbol{\xi}_{2}(k)-\boldsymbol{\alpha}_{2} \\
\left(\boldsymbol{\alpha}_{1}-\boldsymbol{\xi}_{1}(k)\right)+\left(\boldsymbol{\alpha}_{2}-\boldsymbol{\xi}_{2}(k)\right)
\end{array}\right]^{\mathrm{T}}
\end{aligned}
$$


In the system of multi-UAVs cooperative localization, one-step state predicted error is as follows:

$$
\begin{aligned}
\widetilde{\widetilde{\mathbf{x}}}_{k+1, k}= & \widetilde{\mathbf{x}}_{k+1}-\widehat{\widetilde{\mathbf{x}}}_{k+1, k} \\
= & \left(\overline{\boldsymbol{\Phi}}_{k}-\mathbf{L}_{k} \overline{\mathbf{H}}_{k}\right) \widetilde{\widetilde{\mathbf{x}}}_{k, k-1}+\Delta \boldsymbol{\Phi}_{k} \widetilde{\mathbf{x}}_{k}-\mathbf{L}_{k} \Delta \mathbf{H}_{k} \widetilde{\mathbf{x}}_{k} \\
& +\widetilde{\boldsymbol{\Gamma}}_{k} \mathbf{W}_{k}-\mathbf{L}_{k} \boldsymbol{\xi}_{1}(k) \mathbf{V}_{k} .
\end{aligned}
$$

From this formula, we know there was system state in one-step predicted error. So the system state covariance matrix is introduced. We use $\mathbf{q}(t)$ to represent system state covariance, $\mathbf{q}_{k}=E\left[\mathbf{x}_{k} \mathbf{x}_{k}^{\mathrm{T}}\right]$. The next step can be represented as follows:

$$
\begin{aligned}
\mathbf{q}_{k+1}= & \overline{\boldsymbol{\Phi}}_{k} q_{k} \overline{\boldsymbol{\Phi}}_{k}^{\mathrm{T}}+\left[\begin{array}{lll}
0 & 0 & 0 \\
0 & 0 & 0 \\
0 & 0 & \mathbf{A}_{1}
\end{array}\right] \otimes\left[\boldsymbol{\Phi}_{k}^{1} \mathbf{q}_{k} \boldsymbol{\Phi}_{k}^{1^{\mathrm{T}}}\right] \\
& +\left[\begin{array}{lll}
0 & 0 & 0 \\
0 & 0 & 0 \\
0 & 0 & \mathbf{A}_{2}
\end{array}\right] \otimes\left[\boldsymbol{\Phi}_{k}^{2} \mathbf{q}_{k} \boldsymbol{\Phi}_{k}^{2^{T}}\right]+\left[\begin{array}{lll}
0 & 0 & 0 \\
0 & 0 & 0 \\
0 & 0 & \mathbf{A}_{3}
\end{array}\right] \\
& \otimes \mathbf{q}_{k}+\mathbf{Q},
\end{aligned}
$$

where

$$
\begin{aligned}
& \mathbf{A}_{1}=\operatorname{diag}\left(\alpha_{1}^{1}\left(1-\alpha_{1}^{1}\right) \mathbf{1}_{m_{1}}, \ldots, \alpha_{1}^{L}\left(1-\alpha_{1}^{L}\right) \mathbf{1}_{m_{L}}\right), \\
& \mathbf{A}_{2}=\operatorname{diag}\left(\alpha_{2}^{1}\left(1-\alpha_{2}^{1}\right) \mathbf{1}_{m_{1}}, \ldots, \alpha_{2}^{L}\left(1-\alpha_{2}^{L}\right) \mathbf{1}_{m_{L}}\right), \\
& \mathbf{A}_{3}=\operatorname{diag}\left(\left(\alpha_{1}^{1}\left(1-\alpha_{1}^{1}\right)+\alpha_{2}^{1}\left(1-\alpha_{2}^{1}\right)\right)\right. \\
&\left.\cdot \mathbf{1}_{m_{1}}, \ldots,\left(\alpha_{1}^{L}\left(1-\alpha_{1}^{L}\right)+\alpha_{2}^{L}\left(1-\alpha_{2}^{L}\right)\right) \mathbf{1}_{m_{L}}\right), \\
& \boldsymbol{\Phi}_{k}^{1}=\left[\begin{array}{lll}
0 & 0 & 0 \\
0 & 0 & 0 \\
\mathbf{H}_{k} & 0 & 0
\end{array}\right], \\
& \boldsymbol{\Phi}_{k}^{2}=\left[\begin{array}{lll}
0 & 0 & 0 \\
0 & 0 & 0 \\
0 & 1 & 0
\end{array}\right],
\end{aligned}
$$

where $\otimes$ represents Hadamard Product; we define $\mathbf{B} \otimes \mathbf{C}=$ $\left(b_{i j} \times c_{i j}\right)_{r \times s}$, and also

$$
\begin{gathered}
{\left[\begin{array}{cccc}
a_{1} \mathbf{I}_{m_{1}} & & \\
& \ddots & \\
& & a_{k} \mathbf{I}_{m_{k}}
\end{array}\right]_{r \times r}\left(b_{i j}\right)_{r \times s}\left[\begin{array}{ccc}
c_{1} \mathbf{I}_{n_{1}} & & \\
& \ddots & \\
& & c_{l} b_{n_{k}}
\end{array}\right]_{s \times s}} \\
\quad=\left[\begin{array}{ccc}
a_{1} c_{1} \mathbf{1}_{m_{1} n_{1}} & \cdots & a_{1} c_{l} \mathbf{1}_{m_{1} n_{l}} \\
\vdots & \ddots & \vdots \\
& & \\
a_{k} c_{1} \mathbf{1}_{m_{k} n_{1}} & \cdots & a_{k} c_{l} \mathbf{1}_{m_{k} n_{l}}
\end{array}\right]_{s \times s} \otimes\left(b_{i j}\right)_{r \times s},
\end{gathered}
$$

$$
\mathbf{Q}=E\left[\widetilde{\boldsymbol{\Gamma}}_{k} \widetilde{\mathbf{w}}_{k} \widetilde{\mathbf{w}}_{k}^{\mathrm{T}} \widetilde{\boldsymbol{\Gamma}}_{k}^{\mathrm{T}}\right]=\left[\begin{array}{ccc}
\boldsymbol{\Gamma}_{k} \mathbf{Q}_{k} \boldsymbol{\Gamma}_{k}^{\mathrm{T}} & 0 & 0 \\
0 & \mathbf{Q}_{v} & \mathbf{Q}_{v} \boldsymbol{\alpha}_{1} \\
0 & \boldsymbol{\alpha}_{1} \mathbf{Q}_{v} & \boldsymbol{\alpha}_{1} \mathbf{Q}_{v} \boldsymbol{\alpha}_{1}
\end{array}\right]
$$

Based on above fundamental theory, multi-UAVs centralized cooperative localization algorithm based on Kalman filter is presented as follows.

(1) One-step state prediction:

$$
\widehat{\widetilde{\mathbf{x}}}_{k+1, k}=\widetilde{\boldsymbol{\Phi}}_{k} \widehat{\widetilde{\mathbf{x}}}_{k, k} .
$$

(2) Mean square error of one-step prediction:

$$
\begin{aligned}
\mathbf{P}_{k+1, k}= & {\left[\overline{\boldsymbol{\Phi}}_{k}-\mathbf{L}_{k} \overline{\mathbf{H}}_{k}\right] \mathbf{P}_{k, k-1}\left[\overline{\boldsymbol{\Phi}}_{k}-\mathbf{L}_{k} \overline{\mathbf{H}}_{k}\right]^{\mathrm{T}}+\mathbf{Q} } \\
& -\mathbf{L}_{k} \boldsymbol{\alpha}_{1} \mathbf{S}_{k}^{\mathrm{T}} \widetilde{\boldsymbol{\Gamma}}_{k}^{\mathrm{T}}-\widetilde{\boldsymbol{\Gamma}}_{k} \mathbf{S}_{k} \boldsymbol{\alpha}_{1} \mathbf{L}_{k}^{\mathrm{T}}+\Delta \boldsymbol{\Phi}_{k} \mathbf{q}_{k} \Delta \boldsymbol{\Phi}_{k}^{\mathrm{T}} \\
& +\mathbf{L}_{k} \Delta \mathbf{H}_{k} \mathbf{q}_{k} \Delta \mathbf{H}_{k}^{\mathrm{T}} \mathbf{L}_{k}^{\mathrm{T}}-\Delta \boldsymbol{\Phi}_{k} \mathbf{q}_{k} \overline{\mathbf{H}}_{k}^{\mathrm{T}} \mathbf{L}_{k}^{\mathrm{T}} \\
& -\mathbf{L}_{k} \Delta \mathbf{H}_{k} \mathbf{q}_{k} \Delta \boldsymbol{\Phi}_{k}^{\mathrm{T}} .
\end{aligned}
$$

(3) State estimation:

$$
\widehat{\widetilde{\mathbf{x}}}_{k, k}=\widehat{\widetilde{\mathbf{x}}}_{k, k-1}+\mathrm{K}_{k} \boldsymbol{\varepsilon}_{k} .
$$

(4) Filter gain:

$$
\mathbf{K}_{k}=\mathbf{P}_{k, k-1} \overline{\mathbf{H}}_{k}^{\mathrm{T}} \mathbf{Q}_{\varepsilon}^{-1}(k) .
$$

(5) MSE (mean square error) estimation:

$$
\mathbf{P}_{k, k}=\mathbf{P}_{k, k-1}-\mathbf{K}_{k} \mathbf{Q}_{\varepsilon}(k) \mathbf{K}_{k}^{\mathrm{T}},
$$

where

$$
\begin{aligned}
\boldsymbol{\varepsilon}_{k} & =\widetilde{\mathbf{y}}_{k}-\overline{\mathbf{H}}_{k} \widehat{\widetilde{\mathbf{x}}}_{k, k-1} \\
& =\overline{\mathbf{H}}_{k}\left(\widetilde{\mathbf{x}}_{k}-\widetilde{\widetilde{\mathbf{x}}}_{k, k-1}\right)+\Delta \mathbf{H} \widetilde{\mathbf{x}}_{k}+\boldsymbol{\xi}_{1}^{k} \widetilde{\mathbf{v}}_{k}, \\
\mathbf{Q}_{\varepsilon}(k) & =\overline{\mathbf{H}}_{k} \mathbf{P}_{k, k-1} \overline{\mathbf{H}}_{k}^{\mathrm{T}}+\Delta \mathbf{H} \mathbf{q}_{k} \Delta \mathbf{H}^{\mathrm{T}}+\boldsymbol{\alpha}_{1} \mathbf{Q}_{v} \boldsymbol{\alpha}_{1} .
\end{aligned}
$$

$\mathbf{L}_{k}$ means one-step predicted gain matrix:

$$
\mathbf{L}_{k}=\overline{\boldsymbol{\Phi}}_{k} \mathbf{K}_{k} .
$$

The initial value of the filter is $\widehat{\widetilde{\mathbf{x}}}_{0,1}=\left[\begin{array}{lll}\mathbf{x}_{0} & \mathbf{0} & \mathbf{0}\end{array}\right]^{\mathrm{T}}, \mathbf{p}_{0,1}=$ $\left[\begin{array}{ccc}\mathbf{p}_{0} & 0 & 0 \\ \mathbf{0} & 0 & 0 \\ \mathbf{0} & 0 & 0\end{array}\right]$, and based on the initial variable, the filter iterates step by step. The system state $\widehat{\widetilde{\mathbf{x}}}_{k+1, k+1}$ could be calculated. And the target state $\widehat{\mathbf{x}}_{k+1, k+1}=\left[\begin{array}{lll}\mathbf{I}_{n} & \mathbf{0} & \mathbf{0}\end{array}\right] \widehat{\widetilde{\mathbf{x}}}_{k+1, k+1}$ could be calculated.

3.2. Distributed Multi-UAVs Cooperative Localization Algorithm. In distributed multi-UAVs cooperative localization algorithm, each UAV senses the target localization information and dose information filter. Then the filter results are sent to the leader. The key problem of the distributed algorithm is how to mitigate the negative aspect of communication constraints.

Mitigation strategies of packet loss and time delay are designed as follows. 
(1) There are no packet loss and no time delay. In this situation, fusion center uses state estimation and MSE estimation received from followers to fuse directly.

(2) There is no packet loss, but there is time delay. In this situation, fusion center uses one-step predicted state and one-step predicted MSE received from followers as the estimated value of the current state and MSE.

(3) There is packet loss. In this situation, fusion center uses two-step predicted state and two-step predicted MSE received from followers as the estimated value of the current state and MSE.

The above strategies can be presented as formula (22), where $\widehat{\mathbf{x}}_{k, k}^{i}$ is target state estimated by $i$ th UAV at time $k . \mathbf{P}_{k, k}^{i}$ is target state MSE estimated by $i$ th UAV at time $k . \widehat{\mathbf{x}}_{k, k-1}^{i}$ is one-step predicted target state estimated by $i$ th UAV at time $k-1$. $\mathbf{P}_{k, k-1}^{i}$ is one-step target state MSE estimated by $i$ th UAV at time $k-1 . \widehat{\mathbf{x}}_{k, k-2}^{i}$ is two-step predicted target state estimated by $i$ th UAV at time $k-2 . \mathbf{P}_{k, k-2}^{i}$ is two-step target state MSE estimated by $i$ th UAV at time $k-2$. $\xi^{i}$ describes the communication between fusion center and $i$ th UAV. $\xi_{1}^{i}(k)=1$ means fusion center can receive information from $i$ th UAV without time delay at time $k . \xi_{2}^{i}(k)=1$ means fusion center can receive information from $i$ th UAV with time delay at time k. $\xi_{1}^{i}(k)=0$ and $\xi_{2}^{i}(k)=0$ mean fusion center cannot receive information from $i$ th UAV at time $k$

$$
\begin{aligned}
\widehat{\mathbf{x}}_{k}^{i}= & \xi_{1}^{i}(k) \widehat{\mathbf{x}}_{k, k}^{i}+\xi_{2}^{i}(k) \widehat{\mathbf{x}}_{k, k-1}^{i}+\left(1-\xi_{1}^{i}(k)-\xi_{2}^{i}(k)\right) \\
& \times\left(\xi_{1}^{i}(k-1) \widehat{\mathbf{x}}_{k, k-1}^{i}+\left(1-\xi_{1}^{i}(k-1)\right) \widehat{\mathbf{x}}_{k, k-2}^{i}\right), \\
P_{k}^{i}= & \xi_{1}^{i}(k) \mathbf{P}_{k, k}^{i}+\xi_{2}^{i}(k) \mathbf{P}_{k, k-1}^{i}+\left(1-\xi_{1}^{i}(k)-\xi_{2}^{i}(k)\right) \\
& \times\left(\xi_{1}^{i}(k-1) \mathbf{P}_{k, k-1}^{i}+\left(1-\xi_{1}^{i}(k-1)\right) \mathbf{P}_{k, k-2}^{i}\right) .
\end{aligned}
$$

In formula (22), the values of $\xi_{1}^{i}(k)$ and $\xi_{2}^{i}(k)$ are uncertain. So we use Bernoulli random variables to describe them. The probability obeys

$$
\begin{aligned}
& \operatorname{prob}\left(\xi_{1}^{i}(k)=1\right)=\alpha_{1}^{i}(k), \\
& \operatorname{prob}\left(\xi_{2}^{i}(k)=1\right)=\alpha_{2}^{i}(k),
\end{aligned}
$$

where

$$
\begin{aligned}
& 0 \leq \alpha_{1}^{i} \leq 1, \\
& 0<\alpha_{2}^{i} \leq 1, \\
& i=2, \ldots, L .
\end{aligned}
$$

$\alpha_{1}^{i}(k)$ is the probability that the fusion center can receive information from $i$ th UAV without time delay at time $k$. $\alpha_{2}^{i}(k)$ is the probability that the fusion center can receive information from $i$ th UAV with time delay at time $k$

$$
\begin{aligned}
& E\left[\xi_{1}^{i}(k)\right]=\alpha_{1}^{i}(k), E\left[\xi_{2}^{i}(k)\right]=\alpha_{2}^{i}(k), \\
& \widehat{\widehat{\mathbf{x}}}_{k}^{i}=E\left[\xi_{1}^{i}(k) \widehat{\mathbf{x}}_{k, k}^{i}+\xi_{2}^{i}(k) \widehat{\mathbf{x}}_{k, k-1}^{i}\right. \\
& \quad+\left(1-\xi_{1}^{i}(k)-\xi_{2}^{i}(k)\right) \\
&\left.\quad \times\left(\xi_{1}^{i}(k-1) \widehat{\mathbf{x}}_{k, k-1}^{i}+\left(1-\xi_{1}^{i}(k-1)\right) \widehat{\mathbf{x}}_{k, k-2}^{i}\right)\right] \\
& \quad=\alpha_{1}^{i}(k) \widehat{\mathbf{x}}_{k, k}^{i}+\alpha_{2}^{i}(k) \widehat{\mathbf{x}}_{k, k-1}^{i}+\left(1-\alpha_{1}^{i}-\alpha_{2}^{i}\right) \\
& \quad \cdot\left(\alpha_{1}^{i} \widehat{\mathbf{x}}_{k, k-1}^{i}+\left(1-\alpha_{1}^{i}\right) \widehat{\mathbf{x}}_{k, k-2}^{i}\right), \\
& \overline{\mathbf{P}}_{k}^{i}=E_{\left[\xi_{1}^{i}(k) \mathbf{P}_{k, k}^{i}+\xi_{2}^{i}(k) \mathbf{P}_{k, k-1}^{i}\right.} \\
& \quad+\left(1-\xi_{1}^{i}(k)-\xi_{2}^{i}(k)\right) \\
&\left.\quad \times\left(\xi_{1}^{i}(k-1) \mathbf{P}_{k, k-1}^{i}+\left(1-\xi_{1}^{i}(k-1)\right) \mathbf{P}_{k, k-2}^{i}\right)\right] \\
& \quad=\alpha_{1}^{i}(k) \mathbf{P}_{k, k}^{i}+\alpha_{2}^{i}(k) \mathbf{P}_{k, k-1}^{i}+\left(1-\alpha_{1}^{i}-\alpha_{2}^{i}\right) \\
& \quad \cdot\left(\alpha_{1}^{i} P_{k, k-1}^{i}+\left(1-\alpha_{1}^{i}\right) P_{k, k-2}^{i}\right) .
\end{aligned}
$$

Based on formula (26), we design distributed multiUAVs cooperative localization algorithm under unknown communication constraints as follows.

(1) Each UAV updates its own state independently:

(a) the leader updates its own state, which includes state estimation and MSE estimation:

$$
\begin{aligned}
\widehat{\mathbf{x}}_{k}^{1} & =\widehat{\mathbf{x}}_{k, k-1}^{1}+\mathbf{K}_{k}\left(\mathbf{z}_{k}-\mathbf{H}_{k} \widehat{\mathbf{x}}_{k, k-1}^{1}\right), \\
\mathbf{P}_{k}^{1} & =\left[\mathbf{I}_{n}-\mathbf{K}_{k} \mathbf{H}_{k}\right] \mathbf{P}_{k, k-1}^{1} .
\end{aligned}
$$

(b) followers update their own states, which include state estimation, MSE estimation, one-step state prediction, one-step MSE estimation, two-step state prediction, and two-step MSE estimation:

$$
\begin{aligned}
\widehat{\mathbf{x}}_{k}^{i} & =\widehat{\mathbf{x}}_{k, k-1}^{i}+\mathbf{K}_{k}\left(\mathbf{z}_{k}-\mathbf{H}_{k} \widehat{\mathbf{x}}_{k, k-1}^{i}\right), \\
\mathbf{P}_{k}^{i} & =\left[\mathbf{I}_{n}-\mathbf{K}_{k} \mathbf{H}_{k}\right] \mathbf{P}_{k, k-1}^{i},
\end{aligned}
$$

$$
i=2, \ldots, L,
$$

$$
\begin{aligned}
& \widehat{\mathbf{x}}_{k, k-1}^{i}=\boldsymbol{\Phi}_{k-1} \widehat{\mathbf{x}}_{k-1}^{i}, \\
& \mathbf{P}_{k, k-1}^{i}=\boldsymbol{\Phi}_{k-1} \mathbf{P}_{k-1} \boldsymbol{\Phi}_{k-1}^{\mathrm{T}}+\Gamma_{k-1} \mathbf{Q}_{k-1} \Gamma_{k-1}^{\mathrm{T}},
\end{aligned}
$$

$$
i=2, \ldots, L,
$$

$\widehat{\mathbf{x}}_{k, k-2}^{i}=\boldsymbol{\Phi}_{k-1} \widehat{\mathbf{x}}_{k-1, k-2}^{i}$

$\mathbf{P}_{k, k-2}^{i}=\Phi_{k-2} \mathbf{P}_{k-1, k-2}^{i} \Phi_{k-2}^{\mathrm{T}}+\boldsymbol{\Gamma}_{k-2} \mathbf{Q}_{k-2} \Gamma_{k-2}^{\mathrm{T}}$ $i=2, \ldots, L$. 


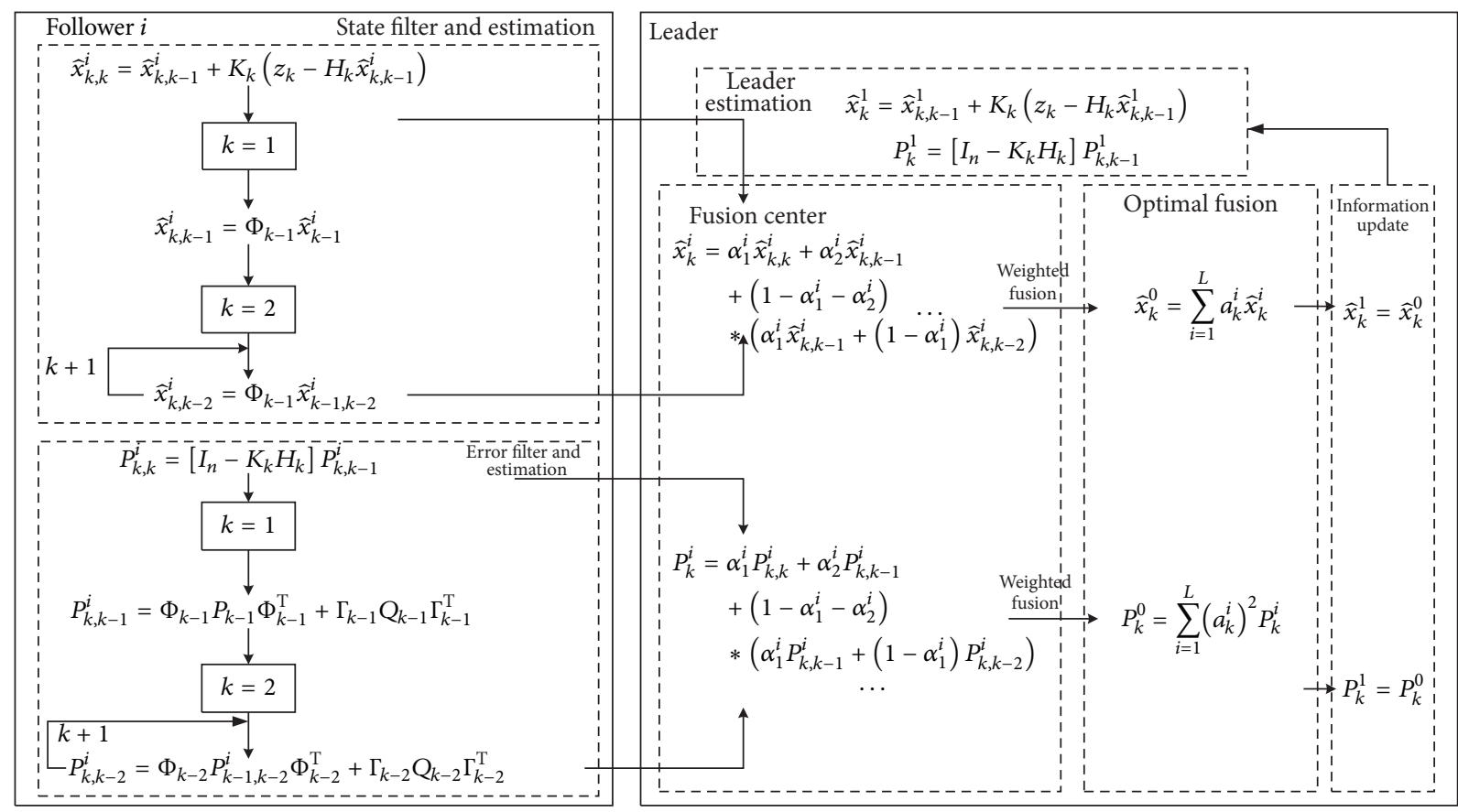

FIGURE 2: The algorithm flow chart of distributed multi-UAVs cooperative localization algorithm.

(2) Fusion center fuses all this information:

(a) received information is compensated by fusion center as follows:

$$
\begin{aligned}
\overline{\widehat{\mathbf{x}}}_{k}^{i}= & \alpha_{1}^{i} \widehat{\mathbf{x}}_{k, k}^{i}+\alpha_{2}^{i} \widehat{\mathbf{x}}_{k, k-1}^{i} \\
& +\left(1-\alpha_{1}^{i}-\alpha_{2}^{i}\right)\left(\alpha_{1}^{i} \widehat{\mathbf{x}}_{k, k-1}^{i}+\left(1-\alpha_{1}^{i}\right) \widehat{\mathbf{x}}_{k, k-2}^{i}\right), \\
\overline{\mathbf{P}}_{k}^{i}= & \alpha_{1}^{i} \mathbf{P}_{k, k}^{i}+\alpha_{2}^{i} \mathbf{P}_{k, k-1}^{i} \\
& +\left(1-\alpha_{1}^{i}-\alpha_{2}^{i}\right)\left(\alpha_{1}^{i} \mathbf{P}_{k, k-1}^{i}+\left(1-\alpha_{1}^{i}\right) \mathbf{P}_{k, k-2}^{i}\right) ;
\end{aligned}
$$

(b) compensated information is weighted by scalars as follows:

$$
\begin{aligned}
& \widehat{\mathbf{x}}_{k}^{0}=\sum_{i=1}^{L} a_{k}^{i} \widehat{\mathbf{x}}_{k}^{i}, \\
& a_{k}^{i}=\left(\sum_{i=1}^{L} \frac{1}{\operatorname{tr} \mathbf{P}_{k}^{i}}\right)^{-1} \frac{1}{\operatorname{tr} \mathbf{P}_{k}^{i}}, \\
& \mathbf{P}_{k}^{0}=\sum_{i=1}^{L}\left(a_{k}^{i}\right)^{2} \mathbf{P}_{k}^{i} .
\end{aligned}
$$

(3) The feedback is from fusion center to the leader UAV.

There are no communication constraints between the fusion center and the leader UAV, so we can add the feedback from the fusion center to the leader UAV to improve state estimation precision of the leader UAV.

$$
\begin{aligned}
\widehat{\mathbf{x}}_{k}^{1} & =\widehat{\mathbf{x}}_{k}^{0}, \\
\mathbf{P}_{k}^{1} & =\mathbf{P}_{k}^{0} .
\end{aligned}
$$

The algorithm flow chart of distributed multi-UAVs cooperative localization algorithm is presented in Figure 2.

\section{Simulation Results}

Two UAVs are used to localize the moving target. Each UAV localizes the moving target by the single station target positioning method. The communicating situation between these two UAVs can be described as follows.

The probability that the fusion center receives target information from follower UAV without time delay is 0.4 , the probability that the fusion center receives target information from follower UAV with 1s time delay is 0.3 , and the probability that the fusion center cannot receive target information from follower UAV is 0.3 .

The targets move with constant velocity along straight trajectory, and its initial state is $\mathbf{X}_{0}=$ $[100,10,100,10,100,0]^{T}$, which means the initial target location is $(100 \mathrm{~m}, 100 \mathrm{~m}, 100 \mathrm{~m})$, and the initial target speed $(10 \mathrm{~m} / \mathrm{s}, 0 \mathrm{~m} / \mathrm{s}, 10 \mathrm{~m} / \mathrm{s})$.

The interference of the UAV localization obeys Gauss distribution of which mathematical expectation is 0 and variance is 10 . 


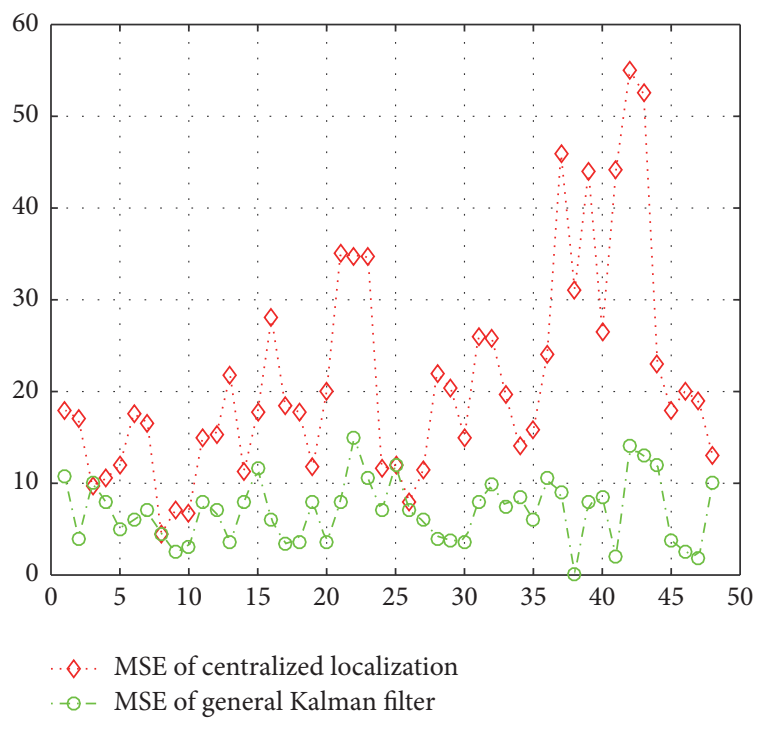

FIgURE 3: The comparison of presented localization algorithm and general Kalman filter algorithm under centralized multi-UAVs cooperative localization.

The process noise of the target motion obeys the Gauss distribution and its mean variance is equal to 5 . The covariance matrix of the initial target localization is set as follows.

$$
P_{0}=\left[\begin{array}{cccccc}
100 & 0 & 0 & 0 & 0 & 0 \\
0 & 5 & 0 & 0 & 0 & 0 \\
0 & 0 & 100 & 0 & 0 & 0 \\
0 & 0 & 0 & 5 & 0 & 0 \\
0 & 0 & 0 & 0 & 100 & 0 \\
0 & 0 & 0 & 0 & 0 & 5
\end{array}\right] .
$$

MSE between the target location and the localization results with communication constraints is shown in Figures 3 and 4 . Figure 3 shows the localization's MSE calculated by centralized localization algorithm, while Figure 4 shows the localization's MSE calculated by distributed localization algorithm.

According to the simulating results, we can figure out that the general Kalman filter algorithm cannot get desired localization results under communication constraints, and it also has convergent problems. The presented multi-UAVs cooperative localization algorithm can get better results under communication constraints.

From Figure 5 we know that the localization results calculated by centralized multiple UAVs cooperation localization algorithm are better than distributed algorithm. But based on the theory of the algorithm's complexity, the complexity of the centralized algorithm is $O\left((n+2 m)^{3}+m^{3}+m(n+2 m)^{2}+\right.$ $\left.(n+2 m) n^{2}\right)$, and the complexity of the distributed algorithm is $O\left(m^{3}+n^{3}+m^{2} n+m n^{2}\right)$, where $n$ and $m$ represent system's state dimension and observation dimension. So distributed algorithm is more appropriate for engineering application.

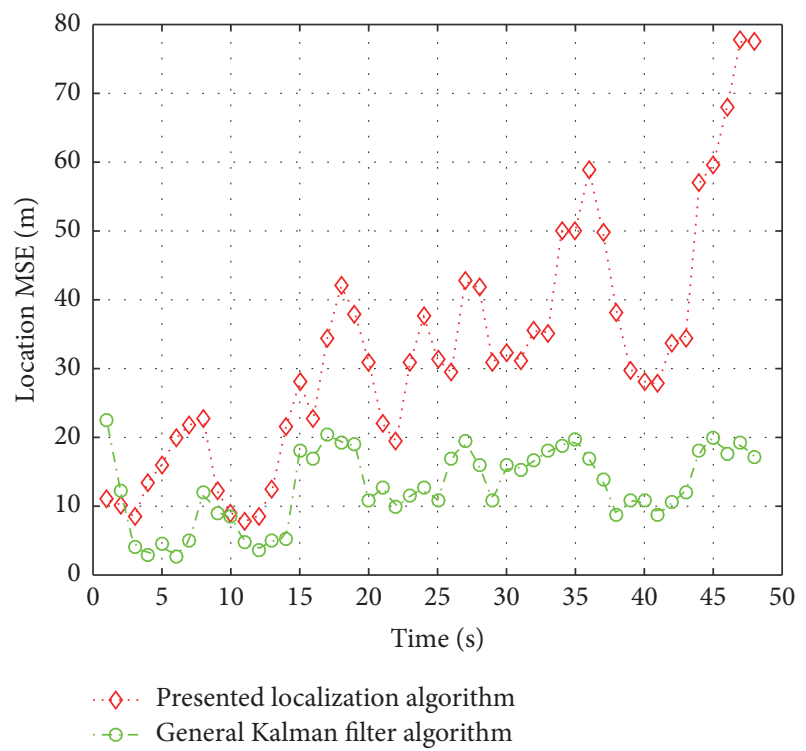

FIGURE 4: The comparison of presented localization algorithm and general Kalman filter algorithm under distributed multi-UAVs cooperative localization.

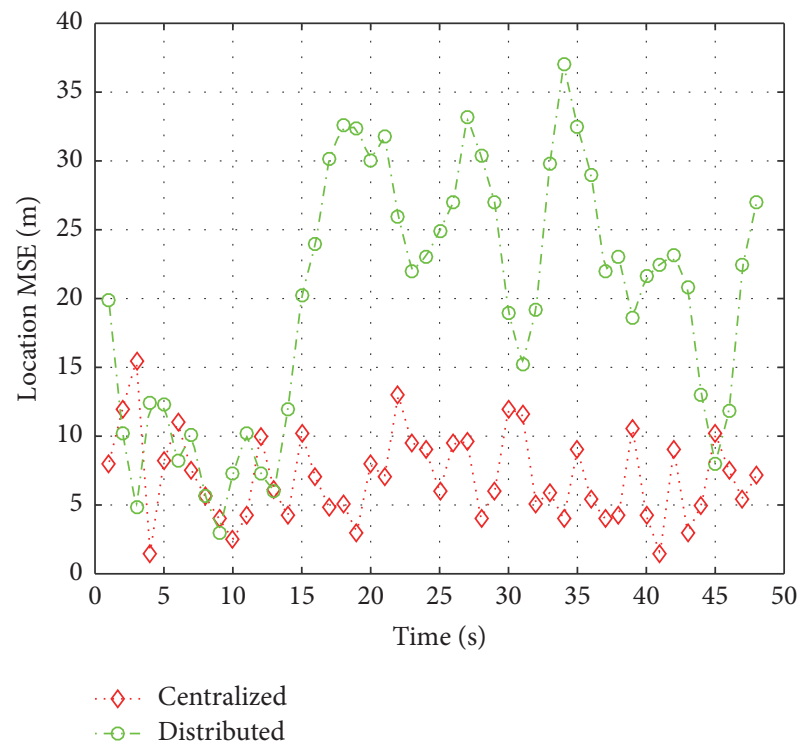

FIGURE 5: The comparison between the distributed and centralized cooperative localization algorithm.

Both centralized and distributed cooperative localization algorithms are IIR filtering approach. Compared to FIR filter approach, such as hybrid particle/FIR filtering [11], composite particle/FIR filtering [12], and deadbeat dissipative FIR filtering [13], the presented algorithms have greater mean square error when there are model uncertainty and/or numerical error in the system, but these proposed algorithms can still meet the needs of practical positioning. And to design filters with the same parameters, FIR approaches need more parameters than these proposed algorithms; this will increase the amount of computation of the DSP. That is to 
say, DSP requires more computation time, and this will have negative impact on the real-time performance of DSP. For the problem of multiple UAVs cooperation localization, the real-time performance of designed algorithm is critical. So the presented algorithms in this paper are a compromise between accuracy and real-time, and these algorithms are more appropriate for multiple UAV s cooperation localization.

\section{Conclusion}

(1) This paper analyzes the problem of multiple UAVs cooperation localization under the condition of communication limits and explains the negative influence of communication latency and information packet loss on the multiple UAVs cooperation localization.

(2) Two kinds of cooperative localization algorithms are presented. In centralized algorithm, extended dimension information fusion is used to establish the state equation with communication latency and information packet loss, and state covariance is considered based on projection theory. In distributed algorithm, a kind of predicting and compensating strategy is designed to deal with communication constraints.

(3) Simulation verifies the proposed algorithms and analyses the complexity and the localization precision of the centralized and distributed algorithm.

\section{Conflicts of Interest}

The authors declare that they have no conflicts of interest.

\section{Acknowledgments}

This work was supported by the Science and Technology on Avionics Integration Laboratory and the Aviation Science Foundation under Grant 20155553041.

\section{References}

[1] A. Chiuso and L. Schenato, "Performance bounds for information fusion strategies in packet-drop networks," in Proceedings of the 2009 European Control Conference (ECC), pp. 1304-1316, 2009.

[2] L. Schenato, "Optimal estimation in networked control systems subject to random delay and packet drop," Institute of Electrical and Electronics Engineers. Transactions on Automatic Control, vol. 53, no. 5, pp. 1311-1317, 2008.

[3] W.-A. Zhang, G. Feng, and L. Yu, "Multi-rate distributed fusion estimation for sensor networks with packet losses," Automatica. A Journal of IFAC, the International Federation of Automatic Control, vol. 48, no. 9, pp. 2016-2028, 2012.

[4] N. Lv and S. Sun, "Scalar-weighted fusion estimators for systems with multiple sensors and multiple delayed measurements," in Proceedings of the IEEE Conference on Decision and Control, pp. 7599-7602, 2010.

[5] R. Rahman, M. Alanyali, and V. Saligrama, "Distributed tracking in multi-hop networks with communication delays and packet losses [C]," in Statistical Signal Processing, pp. 1190-1195, IEEE, Singapore, Asia, 13th edition, 2005.
[6] R. Sanchis and I. Peñarrocha, "Fault detection and estimation in systems with scarce measurements," in Proceedings of the 7th IFAC Symposium on Fault Detection, Supervision and Safety of Technical Processes, pp. 113-118, 2009.

[7] S. C. Smith and P. Seiler, "Estimation with lossy measurement: jump estimators for jump systems," Institute of Electrical and Electronics Engineers. Transactions on Automatic Control, vol. 48, no. 12, pp. 2163-2171, 2003.

[8] J. Ma and S. Sun, "Information fusion estimators for systems with multiple sensors of different packet dropout rates," Information Fusion, vol. 12, no. 3, pp. 213-222, 2011.

[9] J. Ma and S. Sun, "Centralized fusion estimators for multisensor systems with random sensor delays, multiple packet dropouts and uncertain observations," IEEE Sensors Journal, vol. 13, no. 4, pp. 1228-1235, 2013.

[10] S. Sun, L. Xie, W. Xiao, and Y. . Soh, "Optimal linear estimation for systems with multiple packet dropouts," Automatica. A Journal of IFAC, the International Federation of Automatic Control, vol. 44, no. 5, pp. 1333-1342, 2008.

[11] J. M. Pak, C. K. Ahn, P. Shi, Y. S. Shmaliy, and M. T. Lim, "Distributed hybrid particle/FIR filtering for mitigating NLOS effects in TOA based localization using wireless sensor networks," IEEE Transactions on Industrial Electronics, vol. 64, no. 6, pp. 5182-5191, 2017.

[12] J. M. Pak, C. K. Ahn, Y. S. Shmaliy, P. Shi, and M. T. Lim, "Accurate and Reliable Human Localization Using Composite Particle/FIR Filtering," IEEE Transactions on Human-Machine Systems, vol. 47, no. 3, pp. 332-342, 2016.

[13] C. K. Ahn, P. Shi, and M. V. Basin, "Deadbeat dissipative FIR filtering," IEEE Transactions on Circuits and Systems, vol. 63, no. 8, pp. 1210-1221, 2016. 


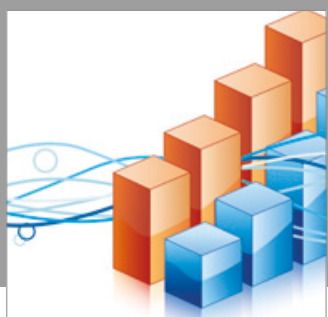

Advances in

Operations Research

vatersals

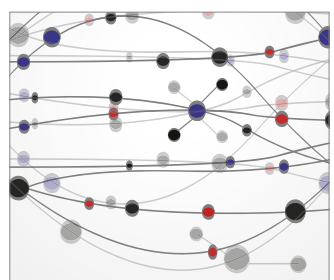

\section{The Scientific} World Journal
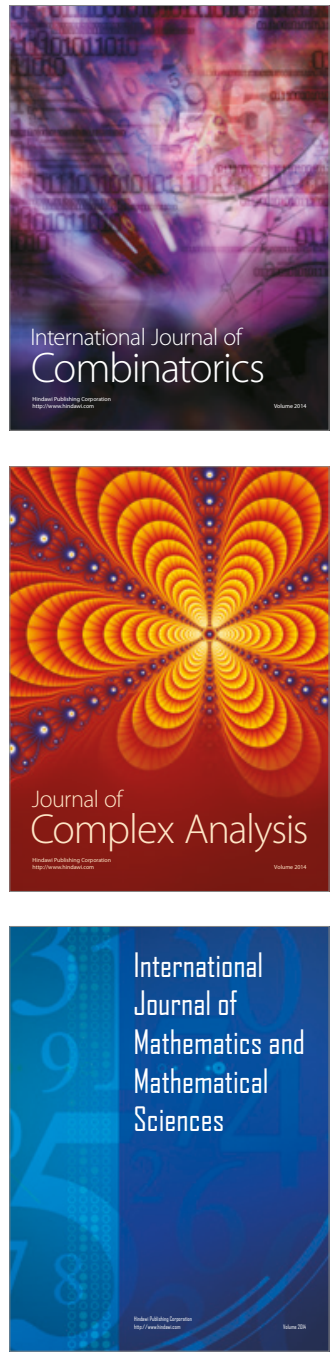
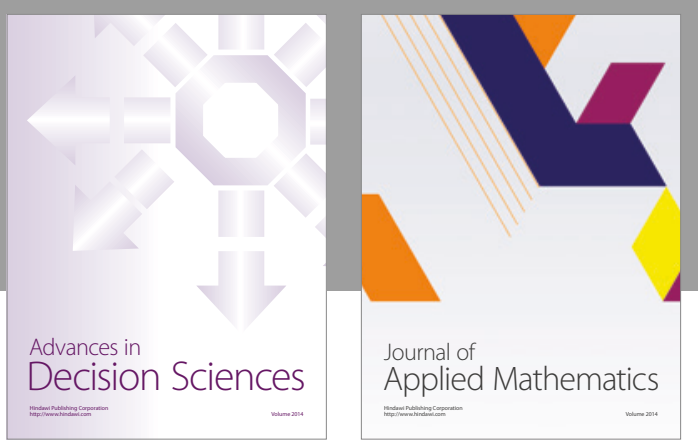

Algebra

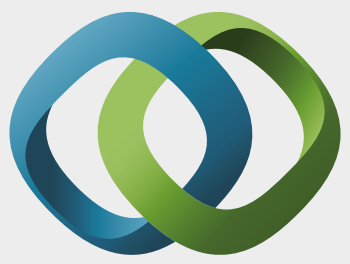

\section{Hindawi}

Submit your manuscripts at

https://www.hindawi.com
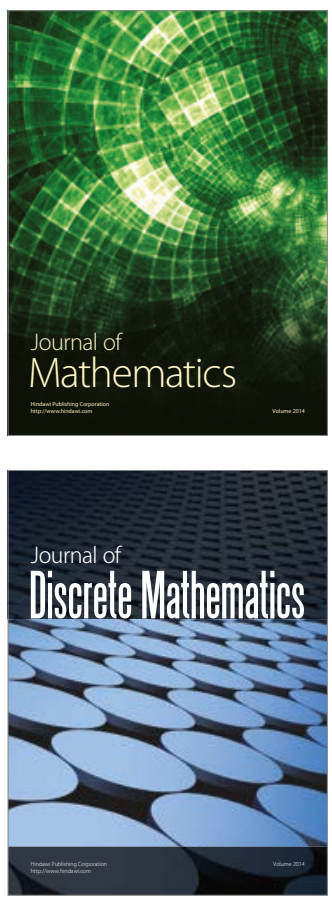

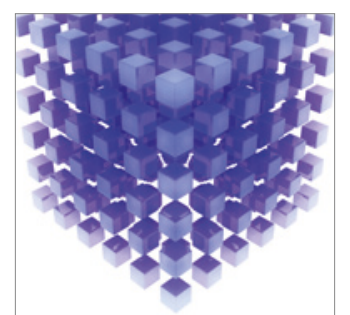

Mathematical Problems in Engineering
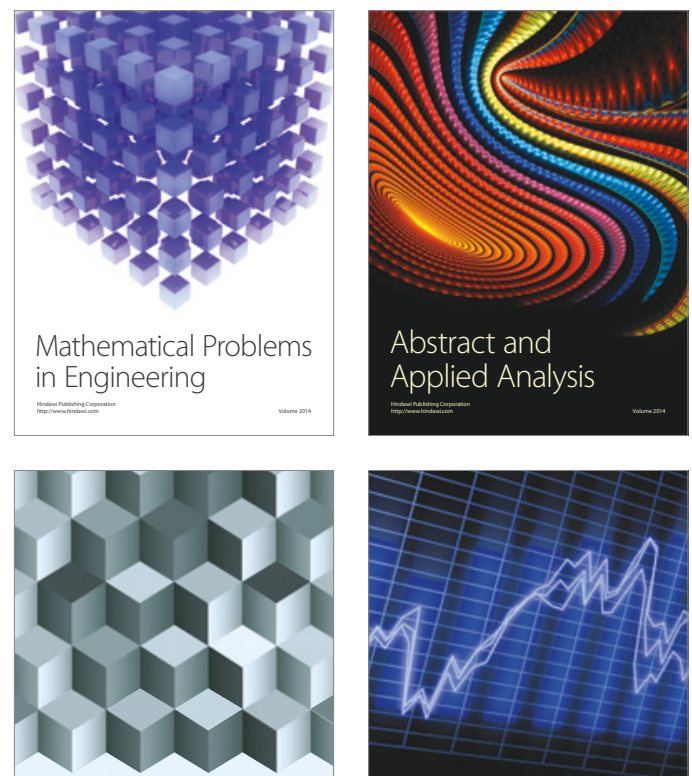

Journal of

Function Spaces

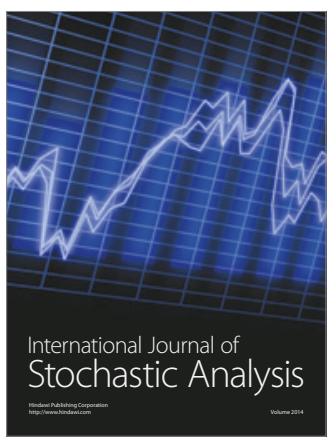

Probability and Statistics
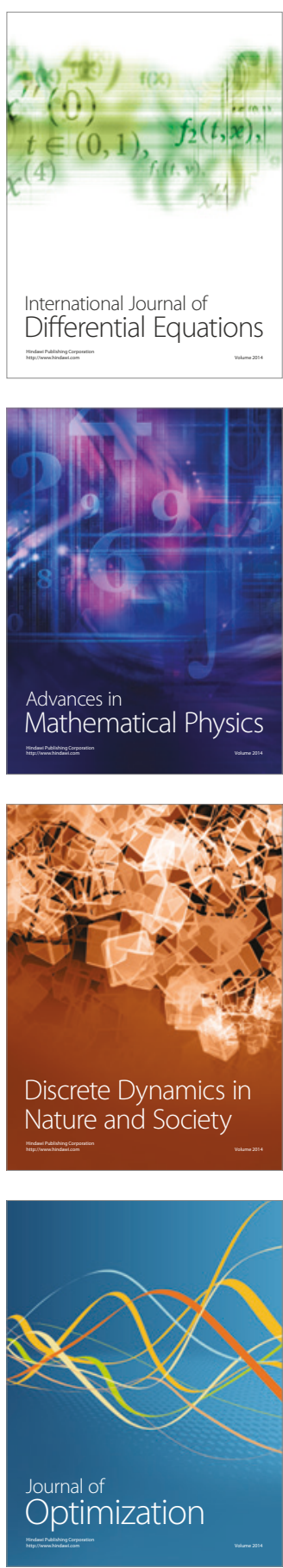